\section{Molecular Structure}

Molecular Structure and Properties. Edited by G. Allen. (MTP International Review of Science.) Pp. 264. (Butterworth: London; University Park: Baltimore, 1972.) $£ 10 ; \$ 24.50$.

THE inevitable growth of the subject and literature has made the part played by reviews in the physical sciences particularly vital. When the field, as here, underlies a large proportion of chemistry and physics, the editor, as Professor Allen points out, faces severe problems in the selection of topics, which must indeed be to some extent subjective. A compromise is made here by the combination of surveys of well established work of continued importance with those of fields which represent growth points. "Neutron Diffraction" (G. E. Bacon), "Electron Diffraction by Gases" (K. Kuchitsu), and "Structure of Crystalline Polymers" (H. Tadokoro) represent long-established areas, while more novel fields are "Acoustic Studies of Molecular Conformational Changes" (A. M. North and R. A. Pethrick), "Magnetic Susceptibilities of Diamagnetic Molecules" (R. Ditchfield), and "Techniques and Uses of Infrared Refractivity" (J. Haigh and L. E. Sutton); related to the last, but curiously separated from it in the text, is a review of the theory of dielectric polarization (A. D. Buckingham).

Tadokoro considers with some systematic formality the results of the study of crystalline polymers, by X-ray and other techniques, during the past five years, in the context of the important problems which remain. Bacon, making no attempt at comprehensiveness in reviewing neutron diffraction, gives an authoritative forward-looking survey with illustrations from typical recent studies, and including molecular spectroscopy by inelastic neutron scattering. Kuchitsu's contribution is notable in that, throughout, it considers the collective evidence of electron diffraction and spectroscopic methods. Although a spectroscopist might question the literal truth of the statement in the preface that electron diffraction remains the major technique for all but the simplest molecules, there is no doubt that the most meaningful treatment of the structures of free molecules involves simultaneous application of diffraction and spectroscopic data. Those seeking the "best" values of geometric parameters in key structures are likely to refer to Kuchitsu here for some time.

Electron diffraction and spectroscopy have provided much information about the geometry and energetics of large amplitude motions within molecules, notably internal rotation or inversion of groups, and changes in ring conformations. The important and largely complementary contributions made by acoustic studies to our knowledge of such motions, particularly when higherenergy conformations are weakly populated, are well brought out by North and Pethrick. The reviews of infrared refractivity and theory of dielectric polarization fittingly emphasise the bridge which must be made between the properties of free molecules and the bulk properties of materials.

The review by Ditchfield of magnetic susceptibilities of diamagnetic molecules is particularly welcome in bringing together aspects of many fields. As befits the current interest in the directional magnetic properties of free molecules, consideration of molecular orbital interpretations is included at some length, while the determination of magnetic anisotropies of molecules from crystal data, Cotton-Mouton studies and, particularly, the Zeeman effect in microwave spectroscopy is discussed along with the evidence of magnetic shielding in n.m.r. Anyone wishing to appreciate the growing contribution of detailed knowledge of molecular magnetism to our critical appraisal of descriptions of structure can start here.

All these chapters, which have been painstakingly produced, will be valuable to structure chemists, and to many who might not describe themselves in precisely that way. When one remembers that volumes 3 and 4 of the same series will concentrate on spectroscopy, one recognizes in the MTP Reviews an unusually well-balanced authoritative publication.

J. SHERIDAN

\section{Pauli's Physics}

Pauli Lectures on Physics. Volume 1. Electrodynamics. Pp. $\mathrm{x}+160$. Volume 2. Optics and the Theory of Electrons. Pp. 159. Volume 3. Thermodynamics and the Kinetic Theory of Gases. Pp. $\mathrm{x}+138$. Volume 4. Statistical Mechanics. Pp. $\mathrm{x}+121$. Volume 5. Wave Mechanics. Pp. xiv +205 . By Wolfgang Pauli. Edited by Charles P. Enz. Translated by H. R. Lewis and S. Margulies. (MIT: Cambridge, Massachusetts and London, March 1973.) \$9.95 each volume.

Wolfgang Pauli was a great theoretical physicist who made many major contributions to the formulation of the key concepts of present-day theoretical physics. It is therefore of considerable interest to see his approach to the presentation of these concepts through the various lecture courses he gave to students at the Swiss Federal Institute of Technology in Zürich.

The five books reviewed here are excellent translations by $S$. Margulies and $H$. R. Lewis of lecture notes taken by different students and collaborators of Pauli. The notes have been carefully edited by C. P. Enz who provides a preface and an appendix to each volume. The former sets out the circumstances in which the notes were made and the latter consists of a series of short technical comments on different parts of the text. A toreword to this series of books by Victor Weisskopf sets out the rationale for this publication of notes of lectures which, it must be recognized, were in general given more than twenty years ago. Briefly, Weisskopf says that "Pauli's way of presenting physics is never out of date", his style "is commensurate to the greatness of its subject in its clarity and impact" and his lectures "show how physical ideas can be presented clearly and in good mathematical form, without being hidden in formalistic expertise". There can be no argument with these views and the notes in these five volumes are remarkably concise presentations of what might be called basic theoretical physics and, as Weisskopf assures us, "they bear the mark of the master in their conceptual structure and their mathematical rigidity".

The level of each course goes rather beyond that of a standard undergraduate physics course bearing the same name in a British university but is probably at about the level found in theoretical physics options. The question naturally arises whether these volumes might be useful as "course" books. Here it is doubtful whether they would be for standard courses. They make no concessions to the reader, explanations are not elaborated and in any case it is assumed implicitly that the reader has been through a significant introductory course beforehand. In general the lectures embark at once on the setting up of a firm mathematical formalism and this style is continued throughout. As his students say in a preface to Volume 5, "Professor Pauli was fond of expressing himself with formulas and only a few words. Once he said 'One should not write so much" ".

That being said, there is no doubt that the books could provide excellent "additional reading" and would be particularly valuable for the wellmotivated and enthusiastic student. Equally they could provide a good basis for optional courses in theoretical physics either for physics or mathematics based undergraduates.

Together they are a remarkable testimony to the elegance of Pauli's approach to physics, his powers of exposition and his penetrating insight. I should certainly wish to associate myself with the concluding sentence of Weisskopf's foreword-"May these volumes serve as an example of how the concepts of theoretical physics were conceived and taught by one of the great men who created them".

\section{R. J. Blin-STOYLE}

